\title{
Advantages of Improved Timing Accuracy in PET Cameras using LSO Scintillator
}

\author{
W. W. Moses, Senior Member, IEEE
}

\begin{abstract}
PET scanners based on LSO have the potential for significantly better coincidence timing resolution than the $6 \mathrm{~ns}$ fwhm typically achieved with BGO. This study analyzes the performance enhancements made possible by improved timing as a function of the coincidence time resolution. If $500 \mathrm{ps}$ fwhm coincidence timing resolution can be achieved in a complete PET camera, the following four benefits can be realized for whole-body FDG imaging: 1) The random event rate can be reduced by using a narrower coincidence timing window, increasing the peak NECR by $\sim 50 \%$. 2) Using time-offlight in the reconstruction algorithm will reduce the noise variance by a factor of 5. 3) Emission and transmission data can be acquired simultaneously, reducing the total scan time. 4) Axial blurring can be reduced by using time-of-flight to determine the correct axial plane that each event originated from. While time-of-flight was extensively studied in the 1980's, practical factors limited its effectiveness at that time and little attention has been paid to timing in PET since then. As these potential improvements are substantial and the advent of LSO PET cameras gives us the means to obtain them without other sacrifices, efforts to improve PET timing should resume after their long dormancy.
\end{abstract}

\section{INTRODUCTION}

I SO scintillator [1] has a number of advantages for PET. LCompared to BGO, it has a similar attenuation length but $4 \mathrm{x}$ higher light output and $7 \mathrm{x}$ shorter scintillation decay time. The higher light output has been exploited to read out a greater number of crystals using a block detector scheme [2]. The shorter decay time implies a reduced dead time, which has allowed quadrant sharing readout schemes (which reduce the number and thus cost of photomultiplier tubes, but increase the effects of dead time) [3]. The faster decay time and higher light output also imply that excellent timing resolution should be possible with LSO, and timing resolutions of $300 \mathrm{ps}$ fwhm have been measured under laboratory conditions [4, 5]. While this resolution was achieved using crystals whose geometry was optimized for timing, resolution slightly better than 500 ps fwhm has been achieved with $3 \times 3 \times 30 \mathrm{~mm}^{3}$ crystal geometries suitable for PET [5]. However, the commercial PET cameras that have been constructed with LSO (the HRRT [6] and the ACCEL) have achieved only 2-3 ns fwhm timing resolution [7]. This resolution is understandable as these cameras are based on

Manuscript received Nov 25, 2002. This work was supported in part by the U.S. Department of Energy under contract No. DE-AC03-76SF00098, and in part by Public Health Service Grants Nos P01-HL25840 and R01CA67911.

W. W. Moses is with Lawrence Berkeley National Laboratory, Berkeley, CA 94720 USA (telephone: 510-486-4432, e-mail: wwmoses@lbl.gov). electronics that were optimized for the relatively poor timing resolution of BGO. This main goal of this paper is to explore what gains in PET performance could be achieved if the timing resolution in an LSO-based PET camera such as the ACCEL were improved.

\section{REDUCED RANDOMS}

One of the major advantages of improved coincidence timing is the reduced random event rate. In PET, the random event rate for an individual chord is given by:

$$
R=2 R_{1} R_{2} \Delta T
$$

where $R$ is the random event rate for that chord, $R_{1}$ and $R_{2}$ are the single event rates for two detector elements that form that chord, and $\Delta T$ is the hardware coincidence timing window width. The total number of random events in the image is the sum over all the chords, thus is proportional to $\Delta T$. The mean contribution to the image from random events can be measured and subtracted, but the noise resulting from the statistical variations in this rate remains.

The practical effect of the residual noise from random coincidences depends on the imaging situation and the task. However, it can be estimated using the noise equivalent count rate (NECR) [8], a common figure of merit for comparing tomograph performance. The NECR is given by:

$$
N E C R=\frac{T^{2}}{T+S+2 R}
$$

where NECR is the noise equivalent count rate, $T$ is the true coincidence event rate, $S$ is the scattered event rate, and $R$ is random event rate. The noise equivalent count (NEC) metric is designed to obey counting statistics; that is, the NEC variance is equal to the NEC. Although the magnitude of the NECR is very sensitive to the source and camera geometries, this formalism is useful for predicting how changes in the trues, randoms, and scatter affect the image quality.

Figure 1 plots, for various hardware coincidence window widths, the expected randoms and trues rates (left) and NECR (right) for a $20 \mathrm{~cm}$ diameter, $20 \mathrm{~cm}$ long phantom imaged in 2-D mode (i.e., with interplane septa) as a function of activity concentration. As the hardware coincidence window width is usually set to twice the coincident timing fwhm (to achieve high efficiency for true events), the data in Figure 1 would be achieved with $6,5,3$, and $2 \mathrm{~ns}$ fwhm coincidence timing resolution. Figure 1 shows that major improvements can be realized just by reducing $\Delta T$, as the randoms depend linearly on the width. Decreasing the width reduces the maximum event rate requirements on the data processing electronics (which are set by the random event rate at high 

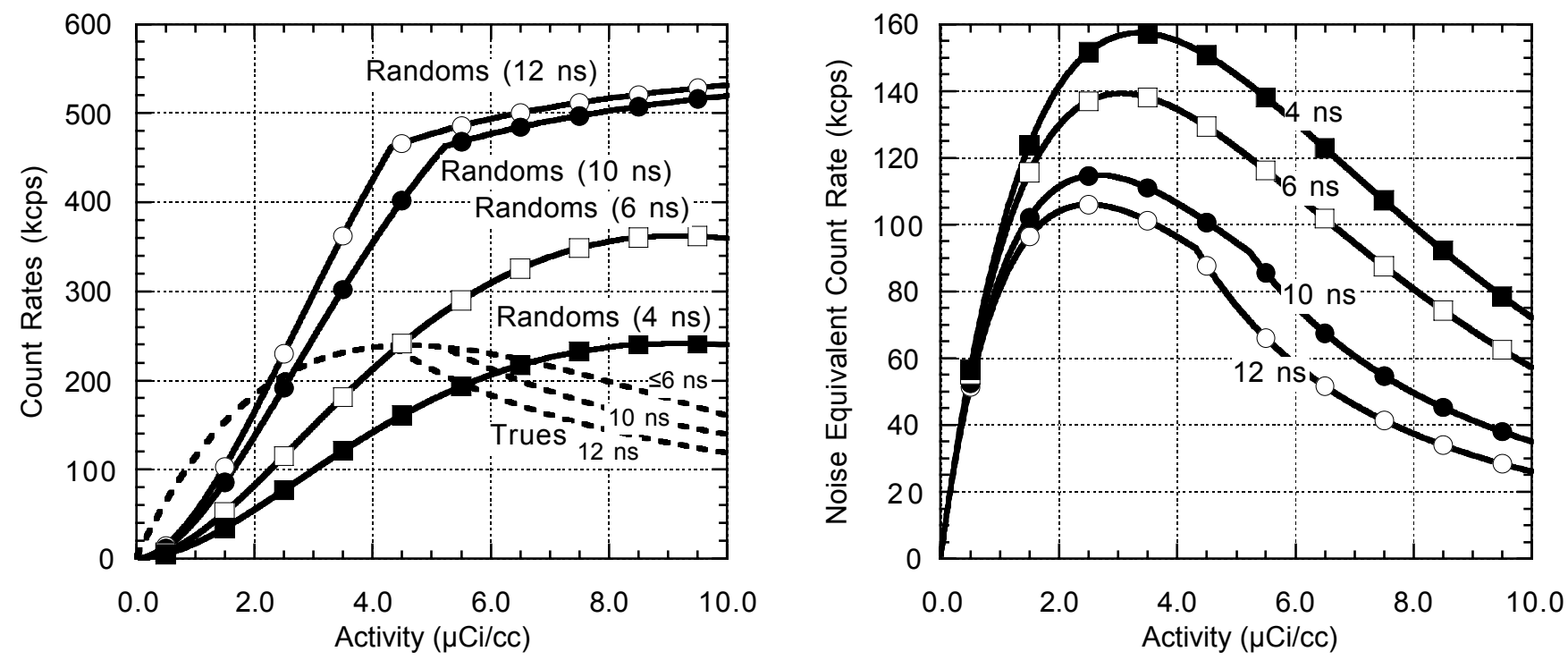

Figure 1: Predicted Randoms and Trues rates (left) and NECR (right) vs. activity concentration and coincidence window width. The object imaged was a uniform $20 \mathrm{~cm}$ diameter cylinder and the camera simulated had an $82 \mathrm{~cm}$ detector ring diameter and $15 \mathrm{~cm}$ axial extent (such as the ECAT EXACT HR).

activity concentrations) and increases the noise equivalent count rate. The peak NECR is predicted to increase from $106 \mathrm{kcps} / \mu \mathrm{Ci} / \mathrm{cc}$ (obtained with a $12 \mathrm{~ns}$ window) to 115 , 139 , and $157 \mathrm{kcps} / \mu \mathrm{Ci} / \mathrm{cc}$ (obtained with a 10,6 , and $4 \mathrm{~ns}$ windows respectively). While this phantom geometry is not the most appropriate for whole-body imaging $(35 \mathrm{~cm}$ diameter with a longer axial extent is more appropriate), it is the most commonly used geometry in the literature and best enables comparison with other PET cameras. Using a larger diameter phantom would shift the curves to the left (i.e., they would peak at lower activity densities). The hardware window width $\Delta T$ cannot be made smaller than $\sim 4 \mathrm{~ns}$, as this is the time-of-flight difference across the detector ring. Reducing the hardware coincidence window below this value might result in valid events near the edge of the field of view being rejected. Thus, the variance reduction due to the number of detected randoms is limited by this $\sim 4 \mathrm{~ns}$ minimum timing window. However, the next section shows that further reduction in the noise variance from random events can be obtained by incorporating time-of-flight information into the reconstruction algorithm.

\section{TIME-OF-FLIGHT RECONSTRUCTION}

\section{A. Theoretical Basis}

Even before computed tomography was applied to positron imaging to create what is now known as PET [9, 10], it was realized that the three-dimensional location of each positron could be directly determined by accurately measuring the difference in arrival times of the two annihilation photons [11]. In other words, the position of the positron would be constrained to a point rather than a line, so three-dimensional images could be obtained without a reconstruction algorithm. The accuracy of the measured position along the line is

$$
\Delta x=\frac{c}{2} \Delta t
$$

where $\Delta x$ is the position error, $c$ is the speed of light, and $\Delta t$ is the error in the timing measurement. To get sub-centimeter position resolution, timing resolution of less than $50 \mathrm{ps}$ is necessary, which is presently impossible to obtain. The achievable timing resolution was $\Delta t \approx 500 \mathrm{ps,} \mathrm{which}$ constrains the positron position to a line segment approximately $7.5 \mathrm{~cm}$ long.

It was realized early in the history of PET that while constraining the positron position to a line segment $7.5 \mathrm{~cm}$ long did not improve the spatial resolution, it did reduce the statistical noise in the reconstructed image if the line segment was shorter than the size of the emission source [12-14]. This multiplicative reduction factor $f$ (corresponding to the reduction in noise variance) is given by

$$
f=\frac{D}{\Delta x}=\frac{2 D}{c \Delta t}
$$

where $D$ is the size of the emission source, $c$ is the speed of light, and $\Delta t$ is the timing error. For organs the size of the brain $(D \approx 20 \mathrm{~cm})$, this factor is greater than unity (implying some noise reduction) for timing resolution $<1.3 \mathrm{~ns}$. For whole-body imaging, the object size is larger $(D \approx 35 \mathrm{~cm})$ and $f>1$ for timing resolution $<2.3$ ns.

The origin of this noise reduction can be understood with the following arguments. With the conventional filtered backprojection algorithm, the fundamental datum is a chord-a line joining the two detector elements that simultaneously observe $511 \mathrm{keV}$ photons. The filtered backprojection algorithm reconstructs an image by backprojecting - incrementing each pixel that lies on that chord by an amount proportional to the number of counts measured in that chord. Placing activity in every pixel along that chord introduces some blurring, but this blurring is removed (modulo statistical noise) by filtering the data before it is backprojected [15].

Reconstruction algorithms for TOF PET were developed in the 1980's and are an adaptation of the filtered backprojection algorithm [13, 16-20]. The main difference is 


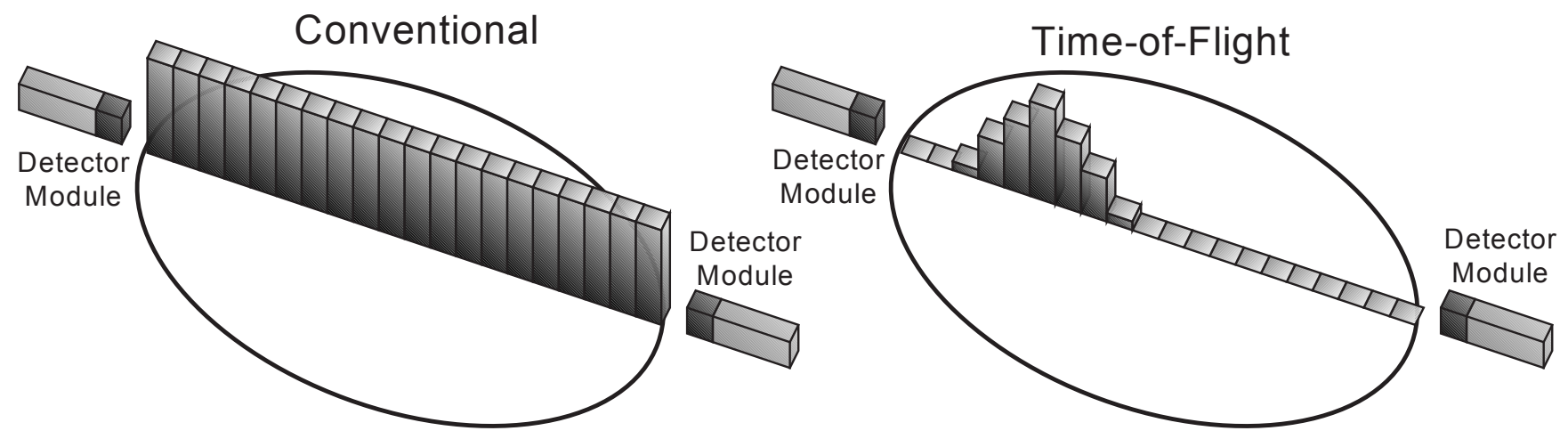

Figure 2: Time-of-Flight Reconstruction. With conventional reconstruction (shown on the left), all pixels along the chord are incremented by the same amount. With time-of-flight reconstruction (shown on the right), each pixel on the chord is incremented by the probability (as determined by the time-offlight measurement) that the source is located at that pixel.

that the fundamental datum consists of both the locations of the two detector elements that observe $511 \mathrm{keV}$ photons and the difference in their arrival times. While this time difference corresponds to a position along the chord, measurement error implies a significant uncertainty in this position. Therefore, not every pixel along that chord is incremented by the same amount when backprojecting. Each pixel is incremented by an amount proportional to the probability (given the measured time difference and the timing resolution) that the annihilation occurred at that pixel, as shown in Figure 2. As with conventional reconstructions, this backprojection introduces some blurring (whose width depends on the timing resolution), but the blurring is removed (modulo statistical noise) by applying an appropriate filter.

Reconstruction algorithms that include time-of-flight information reduce the statistical noise. With non-TOF reconstruction, coincident events measured in a single chord contribute to all of the image pixels along that chord, not just the pixel from which the source truly originated. The reconstruction filter removes the mean contribution to other pixels, but statistical fluctuations in the measurement data cannot be removed and contribute to noise in all the pixels. With time-of-flight reconstruction, coincident events contribute only to those pixels that are near (i.e., are within a distance consistent with the timing resolution) the correct pixel, therefore the statistical fluctuations from the measurement data contribute to a much smaller number of image pixels. This conceptual explanation can be used to derive the variance reduction formula given in Equation 4, and is why time-of-flight can reduce the noise amplification in PET. While the noise reduction is easily predicted for simple, uniform distributions, the improvement depends on the radioisotope distribution, which is rarely homogeneous. However, Equation 4 provides a convenient approximation of the improvement.

The reduction in variance applies not only to the "true" events but also to events that undergo Compton scatter in the patient [21] and to random coincidences [22-25]. This implies that when time-of-flight reconstruction is used, the noise due to randoms continues to diminish as the timing resolution improves, even though the hardware coincidence window is limited by the $\sim 4$ ns minimum set by time-offlight across the patient port. Using the arguments given in the previous paragraph, the effective coincidence window width for computing the noise due to random events (when time-of-flight reconstruction is used) is the time-of-flight measurement resolution, even though the hardware coincidence window is $\geq 4 \mathrm{~ns}$. For random and scattered events, the effective diameter of the emission source (i.e., the diameter of the object that would be reconstructed using just the random or scattered events) is larger than the actual emission source and can be approximated by the camera's patient port diameter. Equation 4 then predicts that the variance reduction will be greater for the random and scatter events than the true events, as their effective source diameters are larger.

The time-of-flight improvement depends strongly on the emission source, so the object must be specified before an accurate estimate of the improvement factor is obtained (we do this in Section IIIB). Estimating the time-of-flight gain is further complicated because the true, scatter, and random events all contribute to noise in the final image and the improvement factor is different for each of these contributions. However, a lower limit on the improvement from TOF reconstruction is easily computed. If we assume that the effective source diameters for the true, random, and scatter events are all equal to the phantom diameter (this is accurate for the trues but underestimates the improvement in randoms and scatter), the variance reduction factors $f$ given by Equation 4 for the trues, the scatters, and the randoms are equal (i.e., $f_{T}=f_{S}=f_{R}=f$ ). As all the variances decrease by the same factor, the noise variance of the final image also decreases by this factor $f$.

\section{B. Measured Improvement}

A number of the TOF PET cameras that were built in the 1980's measured the TOF variance reduction [22, 26-28]. Interpreting these data is difficult, as substantial noise reduction factors often come from three different places: 1) reduced random event rates because the hardware coincidence window is reduced, 2) reduced noise in the true events due to the TOF reconstruction algorithm, and 3) reduced noise in 
the random events due to the TOF reconstruction algorithm [22]. Different authors use different phantom geometries and different activity concentrations, which greatly affects the relative contributions of these factors. While the measured improvement includes contributions from all three of these factors, the TOF literature often attributes the noise reduction in a single factor (usually the trues) rather than estimating the contribution from each of the three factors. Although the NECR metric would have been an excellent framework for estimating the first effect, it was not developed until after research on time-of-flight algorithms had effectively ceased, so the noise reduction due to the lower randoms rate was not quantified in terms of NECR.

For the clinical PET imaging situations most common today, the measurements given in [26] best describe the expected TOF gains. That paper quotes, for a camera with $500 \mathrm{ps}$ resolution, a factor of 3.4 variance reduction for a $35 \mathrm{~cm}$ diameter cylinder, $1.1 \mathrm{~cm}$ thick phantom with $0.12 \mu \mathrm{Ci} / \mathrm{cc}$ activity concentration, which is extremely similar to the source diameter and activity density for a whole-body FDG oncology study $(10 \mathrm{mCi}$ evenly distributed in a $70 \mathrm{~kg}$ patient corresponds to $0.14 \mu \mathrm{Ci} / \mathrm{cc}$ ). Because of the low activity level and short axial extent of the phantom, the contribution from randoms in this measurement is negligible and the factor of 3.4 variance reduction is entirely due to the TOF gain in the true events (this factor is consistent with theoretical predictions). However, the $1.1 \mathrm{~cm}$ axial extent of the phantom causes this measurement to significantly underestimate the contributions from random and scattered events that would be present in a whole-body FDG study. This paper also quotes data with activity concentrations of $0.7 \mu \mathrm{Ci} / \mathrm{cc}$, for which a variance reduction factor of 6.2 is measured [26]. This data better approximates a whole-body FDG study. At this higher activity concentration the contribution from random events is increased, so the improvement due to time-of-flight is larger. As this level of randoms rates is close to what is expected with multi-slice machines, we expect a factor of approximately 6 variance reduction in a modern, whole-body FDG oncology study.

\section{AXIAL BLURRING}

Time-of-flight reconstruction algorithms can improve some aspects of spatial resolution. Timing resolution of $1 \mathrm{~ns}$ fwhm or better can reduce the axial blurring near the edge of the field of view found in reconstruction algorithms that don't accurately place the emission source in the correct plane. Such algorithms include 2-D algorithms that use cross-plane "mashing" [29] (this includes virtually all algorithms used in clinical, whole-body FDG studies) as well as 3-D single slice rebinning (SSRB) [30] algorithms. Because most chords are nearly perpendicular to the central axis of the PET camera, the relatively large uncertainties in the linear distance along the propagation direction (e.g., the $15 \mathrm{~cm}$ implied by $1 \mathrm{~ns}$ timing resolution) have a much smaller axial projection. This allows the time-of-flight algorithm to place events in the correct axial slice with relatively high accuracy and thus reduces the axial blurring (especially near the edge of the field of view) caused by improper "slice" assignment. The improvement factor is equal to the time-of-flight distance uncertainty $(15 \mathrm{~cm}$ for $1 \mathrm{~ns}$ timing uncertainty) divided by the diameter of the emission source ( $\sim 35 \mathrm{~cm}$ for whole-body studies).

\section{SimultaneOUS EMISSION \& TRANSMISSION}

If $\leq 1 \mathrm{~ns}$ fwhm timing resolution is achieved, time-of-flight can separate emission events from transmission events from an external positron source, allowing simultaneous collection of emission and transmission data [31]. A $1 \mathrm{~ns}$ timing uncertainty corresponds to $15 \mathrm{~cm}$ uncertainty in the spatial position of the source, which is approximately the distance between the transmission source and the patient. While one can obtain simultaneous emission and transmission data without time of flight information (by ignoring emission data from chords that are aligned with the transmission source), scattered transmission events generate enough background to the emission data to render the emission data unusable. Time-of-flight information virtually eliminates the scatter contamination, allowing simultaneous collection of emission and transmission data and thus reduced total scanning time.

Some potential drawbacks to this scheme exist. The transmission source(s) can cause significant dead time in the "near" detector modules, reducing their efficiency. In addition, there may be normalization issues. Most PET reconstruction algorithms assume that the efficiency for detecting emissions from a point source placed on a chord is independent of the source's position along that chord, but timing uncertainty may reduce efficiency for locations nearer the edge of the detector ring.

\section{DISCUSSION}

It has long been known that improving the timing performance of PET scanners significantly improves their imaging performance. While the improvement depends on the emission distribution and timing resolution, reductions in the statistical noise variance of a factor of approximately 5 are predicted for objects the size of the (transverse) human body with achievable (500 ps fwhm) timing resolutions. A number of PET cameras incorporating time-of-flight were built in the early 1980's and the predicted gains were observed. However, other performance compromises had to be made to obtain the good timing accuracy that these TOF PET cameras achieved. These sacrifices outweighed the TOF benefits, causing work on time of flight PET to stop around 1990.

Many things have changed in the last decade or two. Of particular importance is the discovery of LSO scintillator, which has demonstrated timing resolutions as good as those achieved with the $\mathrm{BaF}_{2}$ or $\mathrm{CsF}$ scintillators previously used for TOF PET but without the other performance limitations. Equally important is the change in the role of PET. In the 1980's PET cameras had a small axial extent (often a single ring), were used exclusively in a research setting (usually neurology or cardiac studies), and often imaged short-lived 
Table 1: Summary Chart. Estimated benefits as a function of coincidence timing resolution, as compared to a non-TOF BGO-based camera with a $12 \mathrm{~ns}$ coincidence timing window. Object is a uniform $35 \mathrm{~cm}$ diameter phantom.

\begin{tabular}{|l|l|}
\hline Timing Resolution & Performance Gain \\
\hline $2.3-3.0 \mathrm{~ns}$ fwhm & Factor of 1.5-2 reduction in randoms rate. Factor of 1.4-1.6 increase in NECR. \\
\hline $1.5 \mathrm{~ns}$ fwhm & All improvements listed above, plus noise variance decreases by factor of 1.5. \\
\hline $1.0 \mathrm{~ns}$ fwhm & $\begin{array}{l}\text { All improvements listed above, but noise variance decreases by factor of 2.3. Simultaneous } \\
\text { emission/transmission becomes possible. Axial blurring reduced. }\end{array}$ \\
\hline $0.5 \mathrm{~ns}$ fwhm & All improvements listed above, but noise variance decreases by factor of 5. \\
\hline
\end{tabular}

isotopes such as ${ }^{15} \mathrm{O}$ or ${ }^{13} \mathrm{~N}$. Today's PET cameras are wholebody, multi-slice devices that cover a large axial extent, are used for routine diagnosis in high-volume clinical settings, and almost exclusively image the relatively long half-life ${ }^{18} \mathrm{~F}$ isotope. The timing performance of photomultiplier tubes has improved markedly, especially for the small, inexpensive PMTs suitable for PET. Finally, the incredible increase in the capability and accessibility of application-specific integrated circuits (ASICs) enables densities and complexities of PET camera electronics that were unthinkable a decade ago at an astonishingly low cost.

Thus, we believe that it is time to revisit timing resolution for PET [32]. Although many aspects were thoroughly studied many years ago, we believe that the scintillation materials available limited the scope and nature of those studies. Given that LSO-based PET cameras are seeing widespread application in clinical, whole-body imaging, we believe that there is potential for a significant improvement in imaging performance (with little additional cost) merely by fully exploiting LSO's demonstrated timing resolution. The benefits need not be confined to LSO-based cameras. Other new scintillator materials are emerging that posses excellent timing properties, such as LuAP [33, 34] and $\mathrm{LaBr}_{3}$ [35], and PET cameras made with these materials would also enjoy these benefits.

\section{CONCLUSIONS}

The development of LSO scintillator has already given PET cameras improved performance characteristics. However, the excellent timing properties of LSO have not yet been fully exploited in PET. Although timing resolutions below 500 ps fwhm have been demonstrated with single crystals in laboratory conditions, the timing resolution achieved in PET cameras is near an order of magnitude worse. If the timing resolution in PET cameras can be improved, a number of significant improvements can be realized. Probably the most alluring is the large (factor of 5) reduction in noise variance allowed by including time-of-flight into the reconstruction algorithm. Time-of-flight PET was extensively studied in the 1980's and eventually discarded, as other performance tradeoffs imposed by the $\mathrm{CsF}$ and $\mathrm{BaF}_{2}$ scintillator then used for TOF PET outweighed the advantages. LSO has the potential to give the advantages of TOF without the disadvantages, and so it appears that the investigation of time-of-flight PET should resume after its long hiatus.
Performance gains are realized with any improvement in timing resolution and are summarized in Table 1. Even if the resolution is $\geq 2.3 \mathrm{~ns}$, gain is realized from the reduced random event rate. Estimates of this gain are $\sim 15 \%$ increase in peak NECR over the LSO-based ACCEL and $\sim 50 \%$ increase over the BGO-based ECAT EXACT HR. If the resolution is $\leq 2.3 \mathrm{~ns}$, variance reduction from TOF reconstruction is realized with the reduction factor $f$ (given by Equation 4) being inversely proportional to the timing resolution. With timing resolution of $500 \mathrm{ps,} \mathrm{a} \mathrm{variance}$ reduction factor of 5 is achieved. If the resolution is $1 \mathrm{~ns}$ or smaller, simultaneous emission and transmission becomes practical, and spatial resolution gains in the axial direction are obtained.

\section{ACKNOWLEDGMENT}

I would like to thank Dr. Stephen E. Derenzo, Dr. Thomas F. Budinger, Dr. Ronald H. Huesman, and Dr. Jinyi Qi for the many interesting discussions that lead to this work.

This work was supported in part by the Director, Office of Science, Office of Biological and Environmental Research, Medical Science Division of the U.S. Department of Energy under Contract No. DE-AC03-76SF00098, and in part by the National Institutes of Health, National Cancer Institute under grant No. R01-CA67911, and National Institutes of Health, National Heart, Lung, and Blood Institute under grant No. P01-HL25840.

\section{REFERENCES}

[1] C. L. Melcher and J. S. Schweitzer, "Cerium-doped lutetium orthosilicate: a fast, efficient new scintillator," IEEE Trans. Nucl. Sci., vol. NS-39, pp. 502-505, Aug. 1992.

[2] M. E. Casey and R. Nutt, "A multicrystal two dimensional BGO detector system for positron emission tomography," IEEE Trans. Nucl. Sci., vol. NS-33, pp. 460-463, Feb. 1986.

[3] W. H. Wong, J. Uribe, K. Hicks and M. Zambelli, "A 2-dimensional detector decoding study on BGO arrays with quadrant sharing photomultipliers," IEEE Trans. Nucl. Sci., vol. 41, pp. 1453-1457, Aug. 1994.

[4] M. Moszynski, T. Ludziejewski, D. Wolski, W. Klamra and V. V. Avdejcikov, "Timing properties of GSO, LSO and other Ce doped scintillators," Nucl. Instr. Meth., vol. A 372, pp. 51-58, Mar. 1996.

[5] W. W. Moses and S. E. Derenzo, "Prospects for time-of-flight PET using LSO scintillator," IEEE Trans. Nucl Sci., vol. NS-46, pp. 474-478, June 1999.

[6] K. Wienhard, M. Schmand, M. E. Casey, K. Baker, J. Bao, et al., "The ECAT HRRT: performance and first clinical application of the new high resolution research tomograph," IEEE Trans. Nucl. Sci., vol. NS-49, pp. 104-110, Feb. 2002.

[7] M. Schmand, CPS Innovations, Inc. Personal communication, 2002. 
[8] S. C. Strother, M. E. Casey, and E. J. Hoffman, "Measuring PET scanner sensitivity: Relating count rates to image signal-to-noise ratios using noise equivalent counts," IEEE Trans. Nucl. Sci., vol. NS-37, pp. 783-788, Apr. 1990.

[9] J. S. Robertson, R. B. Marr, M. Rosenblum, V. Radeka and Y. L. Yamamoto, "32-Crystal positron transverse section detector," in Tomographic Imaging in Nuclear Medicine, G. S. Freedman, Ed. New York: Society of Nuclear Medicine Press, 1973.

[10] M. M. Ter-Pogossian, M. E. Phelps, E. J. Hoffman and N. Mullani, “A positron emission transaxial tomograph for nuclear imaging (PETT)," Radiology, vol. 114, pp. 89-96, Jan. 1975.

[11] H. O. Anger, "Survey of radioisotope cameras," ISA Transactions, vol. 5, pp. 311-334, 1966.

[12] R. E. Campagnolo, P. Garderet, and J. Vacher, "Tomographie par emetreurs positrons avec mesure de temp de vol," in Communication an Colloque National sur le Traitement du Signal. Nice, France, 1979.

[13] D. L. Snyder, L. J. Thomas, and M. M. Ter-Pogossian, "A mathematical model for positron-emission tomography systems having time-of-flight measurements," IEEE Trans. Nucl. Sci., vol. NS-28, pp. 3575-3583, June 1981.

[14] T. Tomitani, "Image reconstruction and noise evaluation in photon time-of-flight assisted positron emission tomography," IEEE Trans. Nucl. Sci., vol. NS-28, pp. 4582-4589, Dec. 1981.

[15] G. N. Ramachandran and A. V. Lakshminarayanan, "Threedimensional reconstruction from radiographs and electron micrographs: application of convolutions instead of Fourier transforms," Proc. Nat. Acad. Sci., vol. 68, pp. 2236-2240, Sep. 1971.

[16] D. L. Snyder and D. G. Politte, "Image reconstruction from list-mode data in an emission tomography system having time-of-flight measurements," IEEE Trans. Nucl. Sci., vol. NS-30, pp. 1843-1849, June 1983.

[17] D. G. Politte and D. L. Snyder, "Results of a comparative study of a reconstruction procedure for producing improved estimates of radioactivity distributions in time-of-flight emission tomography," IEEE Trans. Nucl. Sci., vol. NS-31, pp. 614-619, Feb. 1984.

[18] D. G. Politte, G. R. Hoffman, D. E. Beecher, D. C. Ficke, T. J. Holmes, et al., "Image-reconstruction of data from Super PETT I: a first-generation time-of-flight positron-emission tomograph," IEEE Trans. Nucl. Sci., vol. NS-33, pp. 428-434, Feb. 1986.

[19] K. Ishii, H. Orihara, and T. Matsuzawa, "Construction function for three-dimensional sinograms of the time-of-flight positron emission tomograph," Rev. Sci. Instr., vol. 58, pp. 1699-1701, Sep. 1987.

[20] M. Yamamoto, N. Nohara, E. Tanaka, T. Tomitani, H. Murayama, et al., "Time-of-flight positron imaging and the resolution improvement by an iterative method," IEEE Trans. Nucl. Sci., vol. NS-36, pp. 9981002, Feb. 1989.

[21] B. Bendriem, F. Soussaline, B. Campagnolo, B. Verrey, P. Wajnberg, et al., "A technique for the correction of scattered radiation in a PET system using time-of-flight information," J. Comput. Assist. Tomogr., vol. 10, pp. 287-95, Mar. 1986.

[22] M. Yamamoto, G. R. Hoffman, D. C. Ficke and M. M. Ter-Pogossian. Imaging algorithm and image quality in time-of-flight assisted positron computed tomography: SUPER PETT I. Proceedings of The Workshop on Time of Flight Tomography, pp. 125-129, (Edited by St. Louis, MO, May 1982.

[23] T. J. Holmes, D. L. Snyder, and D. C. Ficke, "The effect of accidental coincidences in time-of-flight positron emission tomography," IEEE Trans. Med. Img., vol. MI-3, pp. 68-79, June 1984.

[24] T. J. Holmes, D. C. Ficke, and D. L. Snyder, "Modeling of accidental coincidences in both conventional and time-of-flight positron-emission tomography," IEEE Trans. Nucl. Sci., vol. NS-31, pp. 627-631, Feb. 1984.

[25] D. G. Politte and D. L. Snyder, "Corrections for accidental coincidences and attenuation in maximum-likelihood image reconstruction for positron-emission tomography," IEEE Trans. Med. Img., vol. 10, pp. 82-89, Mar. 1991.

[26] M. Yamamoto, D. C. Ficke, and M. M. Ter-Pogossian, "Experimental assessment of the gain achieved by the utilization of time-of-flight information in a positron emission tomograph (Super PETT I)," IEEE Trans. Med. Img., vol. MI-1, pp. 187-192, Sep. 1982.

[27] D. L. Snyder, "Some noise comparisons of data-collection arrays for emission tomography-systems having time-of-flight measurements," IEEE Trans. Nucl. Sci., vol. NS-29, pp. 1029-1033, Feb. 1982.

[28] T. K. Lewellen, R. L. Harrison, and A. N. Bice, "An experimental evaluation of the effect of time-of-flight information in image reconstructions for the Scanditronix/PETT Electronics SP-3000 positron emission tomograph-preliminary results," IEEE Trans. Nucl. Sci., vol. NS-36, pp. 1095-1099, Feb. 1989.

[29] M. Defrise, P. E. Kinahan, D. W. Townsend, C. Michel, M. Sibomana, et al., "Exact and approximate rebinning algorithms for 3-D PET data," IEEE Trans. Med. Img., vol. 16, pp. 145-158, Apr. 1997.

[30] M. E. Daube-Witherspoon and G. Muehllehner, "Treatment of axial data in three-dimensional PET," J. Nucl. Med., vol. 28, pp. 1717-1724, Nov. 1987.

[31] M. Yamamoto, D. C. Ficke, and M. M. Ter-Pogossian, "Effect of the software coincidence timing window in time-of-flight assisted positron emission tomography," IEEE Trans. Nucl. Sci., vol. NS-30, pp. 711714, Feb. 1983.

[32] W. W. Moses, "Potential uses for improved coincidence timing accuracy in PET," J. Nucl. Med., vol. 43, pp. 229P, May 2002.

[33] A. Lempicki, M. H. Randles, D. Wisniewski, M. Balcerzyk, C. Brecher, et al., "LuAlO 3 :Ce and other aluminate scintillators," IEEE Trans. Nucl. Sci., vol. NS-42, pp. 280-284, Aug. 1995.

[34] W. W. Moses, S. E. Derenzo, A. Fyodorov, M. Korzhik, A. Gektin, et al., "LuAlO $\mathrm{Al}_{3} \mathrm{Ce}-\mathrm{a}$ high density, high speed scintillator for gamma detection," IEEE Trans. Nucl. Sci., vol. NS-42, pp. 275-279, Aug. 1995.

[35] E. V. D. van Loef, P. Dorenbos, C. W. E. van Eijk, K. Kramer and H. U. Gudel, "High-energy-resolution scintillator: $\mathrm{Ce}^{3+}$ activated LaBr 3 ," Appl. Phys. Lett., vol. 79, pp. 1573-1575, Sep. 2001. 